\title{
Endoscopic Hemostasis for Non-Variceal Upper Gastrointestinal Bleeding: New Frontiers
}

\author{
Adam Kichler and Sunguk Jang \\ Department of Gastroenterology and Hepatology, Digestive Disease Institute, Cleveland Clinic, Cleveland, OH, USA
}

Non-variceal upper gastrointestinal bleeding (NVUGIB) refers to blood loss from the gastrointestinal tract proximal to the ligament of Treitz due to lesions that are non-variceal in origin. The distinction of the bleeding source as non-variceal is important in numerous aspects, but none more so than endoscopic approaches for successful hemostasis. When a patient presents with acute overt blood loss, NVUGIB is a medical emergency, which requires immediate intervention. There have been major strides in pharmacologic and endoscopic interventions for successful induction and remission of hemostasis in the last two decades. Despite achieving tangible improvements, the burden of the disease and the consequent mortality remain high. To address endoscopic outcomes better, several new technologies have emerged and have been subsequently incorporated to the armamentarium of hemostatic tools. This study aims to provide a succinct review on novel technologies for endoscopic hemostasis. Clin Endosc 2019;52:401-406

Key Words: Endoscopy; Hemostasis; Peptic ulcer; Gastrointestinal hemorrhage

\section{INTRODUCTION}

Acute upper gastrointestinal bleeding (AUGIB) is a common medical condition, which continues to yield significant healthcare burden. Despite major strides in its management, mortality due to all forms of AUGIB remains approximately $10 \% .{ }^{1}$ AUGIB is defined as hemorrhage that originates proximal to the ligament of Treitz and can be further categorized on the basis of its source, i.e., variceal or non-variceal (non-variceal upper gastrointestinal bleeding [NVUGIB]). Despite the decline in its incidence and prevalence over the last two decades, peptic ulcer bleeding (PUB) remains the single most common cause of NVUGIB, accounting for $25 \%-67 \%$ of NVUGIB cases. ${ }^{2}$ According to the Nationwide Inpatient Sam-

Received: June 12, 2018 Revised: February 27, 2019

Accepted: March 16, 2019

Correspondence: Sunguk Jang

Department of Gastroenterology and Hepatology, Digestive Disease Institute, Cleveland Clinic, 9500 Euclid Avenue, Cleveland, OH 44195, USA

Tel: +1-216-636-5444, Fax: +1-216-444-6284, E-mail: jangs@ccf.org ORCID: https://orcid.org/0000-0001-9837-0322

cc This is an Open Access article distributed under the terms of the Creative Commons Attribution Non-Commercial License (http://creativecommons.org/ licenses/by-nc/3.0) which permits unrestricted non-commercial use, distribution, and reproduction in any medium, provided the original work is properly cited. ple, the largest inpatient care database in the United States, the incidence of NVUGIB has shown a steady decline over the last two decades, from 108 to 78 cases per 100,000 individuals. ${ }^{3}$ The in-patient mortality due to NVUGIB also steadily declined in the same period: from $4.5 \%$ in the late 1980 s to $2.1 \%$ in the 2000s. ${ }^{3}$ The acceptance of evidence-based approach of aggressive hemodynamic resuscitation, early administration of proton pump inhibitors (PPIs), and improved efficacy of endoscopic hemostasis have contributed to this positive outcome. The adoption and inclusion of these elements have contributed to the overall improvement in successful management of NVUGIB. For instance, early institution of PPIs at the time of index presentation has effectively diminished the risk of re-bleeding, whereas the use of evidence-based approach in selecting hemostatic methods has increased the success rate of endoscopic intervention. ${ }^{4,5}$

A successful endoscopic hemostasis depends not only on the procedural proficiency of performing endoscopists but also on the hemostatic tools available at their disposal to achieve the desired outcome. Currently, the methods for achieving endoscopic hemostasis are categorized as injectant-, thermal-, or mechanical-based. Numerous studies have reported comparable rates of efficacy between thermal 
and mechanical hemostatic tools, whereas injectant-based treatment as a single modality is no longer being advocated. ${ }^{6}$ Several novel technologies such as new mechanical hemoclips, non-contact pharmacologic hemostatic agents, and new thermal hemostasis devices have emerged in the last decade to augment the endeavor of endoscopic hemostasis. The purpose of this review is to identify some of the newer technologies and compare them with some of the previously available options.

\section{INJECTION THERAPY}

Injection of dilute epinephrine and normal saline is commonly included in the endoscopic management strategy for NVUGIB. The therapeutic mechanism of action of these injectable agents is believed to result from their tamponade effect on surrounding tissues, rather than from the vasoactive properties of epinephrine. For the management of PUB, limited evidence suggests that when compared with injection of smaller volumes $(<10 \mathrm{~mL})$, injection of large volumes (13-20 $\mathrm{mL}$ ) of dilute epinephrine at the time of endoscopic intervention will decrease the rates of recurrent bleeding. ${ }^{8}$ Despite this finding, injection monotherapy is not recommended, as several meta-analyses have shown that strategies for hemostasis that combine injection with either mechanical or thermal therapy are more successful than injection alone. ${ }^{6,9-11}$ Additional agents such as cyanoacrylate glues, thrombin, and fibrin are available; however, these are not frequently utilized in the treatment of NVUGIB.

\section{MECHANICAL THERAPY}

Utilization of devices to impart physical hemostasis has been a mainstay in conventional endoscopic therapy for NVUGIB. ${ }^{12}$ Such devices largely include hemostatic clips and to a lesser extent band ligation tools. The design of these endoscopic hemostatic clips has evolved over the past years, which continues to reflect their intended function of creating mechanical compression in high-risk lesions and those that are actively bleeding. The key features of ideal hemostatic clips include being large enough to span the edges of the lesions, strong enough to appose them firmly, and able to open, close, and rotate to ensure optimal position prior to their deployment. Since the first report in 1989, traditional through-thescope (TTS) metal clips have been proven to be useful for the treatment of numerous types of gastrointestinal lesions once deployed over a bleeding site or at a focal region of a high-risk lesion. ${ }^{13}$ There are numerous industry providers of TTS clips that vary in their rotational capabilities, jaw design, and width. When used alone or in combination with injection therapy, these clips yield greater success in achieving hemostasis than injection therapy alone. ${ }^{9,14}$

There have been several recent advances in the design and functionality of TTS clips that are currently available for use. The Quick Clip 2 and Quick Clip Pro marketed by Olympus (Tokyo, Japan) are available in multiple dimensions, have the capability to open and close allowing appropriate positioning, and possess a 1:1 rotation mechanism. The Boston Scientific Co. (Natick, MA, USA) has developed the 11-mm-wide Resolution Clip engineered to be opened and closed up to five times prior to deployment. The Resolution Clip is capable of generating a maximal pressure of $>200 \mathrm{~mm} \mathrm{Hg}$ in $10 \%$ of training models and has been shown to remain at the deployment site for a longer duration when compared with other clips. Cook Medical (Winston-Salem, NC, USA) currently distributes large Instinct Clips, which have the largest jaw width on the market while retaining their rotational capability. Cook Medical has also developed the uniquely designed three-pronged Tri-Clip; while it certainly is a novel idea, the three-pronged design failed to generate enough force to close fibrotic ulcers and achieved lower rates of hemostasis (94\% vs. 76\%) than did other TTS hemoclips. ${ }^{15}$ The Clipmaster is a clip system developed by Medwork (Aisch, Germany), which consists of three preloaded 360-degree rotatable 12-mm clips in one applicator $(2.6 \mathrm{~mm})$. This allows for all three clips to be deployed in succession without the need to remove the application catheter.

For larger lesions that continue to bleed and are refractory to initial attempts with TTS clips and thermal therapy, overthe-scope clips (OTSCs), such as the Ovesco Clip (Ovesco, Tübingen, Germany) and the Padlock Clip (US endoscopy; Steris, Mentor, OH, USA), have been shown to play a valuable role. ${ }^{16}$ In addition to yielding successful hemostasis, OTSCs are frequently utilized in the closure of larger mucosal defects and fistulous opening. The Ovesco OTSC is a nitinol (Nickel Titanium Polymer) clip, which consists of an externally mounted device associated with an applicator cap affixed to the scope tip, and has been shown to generate a maximal vessel compression of $>200 \mathrm{~mm} \mathrm{Hg}$ in up to $59 \%$ cases. $^{17}$ Once properly positioned, the targeted area can be suctioned into the cap or grasped with forceps and then ultimately clasped after the firing mechanism is activated in a manner similar to that of a variceal banding kit that occupies the working channel of the endoscope. While the data are limited, the existing evidence has shown that the Ovesco OTSC has been effective in achieving hemostasis in individuals who have recurrent or persistent bleeding despite initial attempts of standard injection and hemostatic clip use. ${ }^{16}$ Manta et al. have demonstrat- 
ed that in a series of 30 patients, successful hemostasis was achieved using an OTSC in 97\% of the patients who had previously failed conventional endoscopic therapy. ${ }^{18}$ In comparison to the Bear Trap configuration of the Ovesco, the Padlock clip is star-shaped and is deployed via a firing mechanism that remains outside the endoscope in a parallel manner, thereby leaving the working channel vacant.

\section{THERMAL THERAPY}

There are multiple approaches to thermal therapy to achieve hemostasis, and there have been recent advances as well. The established thermal modalities are traditionally categorized as either contact or non-contact techniques. The contact thermal therapy techniques include the use of electrocautery (multipolar/bipolar) and heater probe devices, which work through the process of co-aptive coagulation in either the preferred head on approach or a tangential manner. Co-aptive coagulation refers to the combination of the first application of mechanical pressure to the bleeding site and the delivery of either an electrical current or heat to coagulate the blood vessel. Non-contact approaches to thermal hemostasis include argon plasma coagulation (APC) and laser phototherapy and function in the absence of direct contact. ${ }^{6}$

With respect to the electrocautery techniques, multipolar or bipolar approaches are utilized preferentially, as they are believed to be safer and more cost-effective. With respect to safety, multipolar electrocoagulation (MPEC) and bipolar cautery devices deliver energy within a fixed circuit and will cease once the temperature of the desiccated tissue reaches $100^{\circ} \mathrm{C}$, thereby limiting the depth of tissue injury with rare occurrences of perforation. ${ }^{19}$ In contrast, heater probes deliver a constant temperature $\left(250^{\circ} \mathrm{C}\right)$ for a predetermined duration and will not cease until the set amount of energy is delivered. Thus, heater probes can cause unpredictable depths of injury and result in higher rates of perforation with estimates ranging up to $3 \%$ of uses. ${ }^{19,20}$ MPEC has also gained increasing popularity owing to its ease of use; the electric current is delivered locally, and there is no need for patient grounding, as required in heater probe devices. With respect to the cost per device, the cost of the currently available MPEC probes ranges from $\$ 230$ to $\$ 335$, while that of the available heater probes is greater than $\$ 500$ each.

APC is a non-contact modality of achieving thermal hemostasis, which utilizes the electrical conductivity of argon gas. Argon gas flows through the treatment catheter at a rate of approximately $1.5-2 \mathrm{~L} / \mathrm{min}$ and is energized, creating a plas$\mathrm{ma}$ arc. The catheter tip should be positioned $2-10 \mathrm{~mm}$ from the mucosal surface prior to activation, and when initiated, the device can cause a superficial $(2-3 \mathrm{~mm})$ thermal injury. Given its superficial nature, APC is believed to be the preferred modality for the treatment of shallow vascular lesions, such as gastric antral vascular ectasia (GAVE), arteriovenous malformation (AVM), and angioectasia. ${ }^{21,22}$ Another, but less commonly utilized form of non-contact thermal therapy, is laser phototherapy. Its mechanism of hemostasis following the injection of dilute epinephrine utilizes an Nd:YAG laser to target the areas surrounding the bleeding lesion. The application of the laser will generate heat, causing vessel wall edema and ultimate hemostasis.

A more recently introduced modality for thermal therapy is the implementation of coagulation forceps. Referred to as soft coagulation, the use of hemostatic forceps with the assistance of a monopolar electrocoagulation system has been proven to be a safe and effective method for achieving hemostasis. With the hemostatic forceps, the bleeding lesion or vessel can be grasped, and once heat is applied, raising the temperature just below the boiling point, tissue dehydration and protein denaturation will occur, creating a functional seal. ${ }^{23}$ This technique has been reported to stop ulcer bleeding successfully in case series and has superiority to the use of heater probes in achieving successful hemostasis. ${ }^{24-27}$

Radiofrequency ablation (RFA), which is currently gaining popularity as a hemostatic method, is another technology established for the management of Barrett's esophagus. Particularly in its application for GAVE, RFA generates a uniform superficial thermal injury, which has been shown to successfully stop bleeding and subsequently minimize transfusion requirements. RFA has also been shown to allow transfusion independence in 18 of 24 patients with transfusion-dependent GAVE in an open-label prospective cohort study. ${ }^{28}$ In a retrospective case series including 24 patients with GAVE who underwent RFA, $63 \%$ of the patients were able to become transfusion-independent at 6 months. ${ }^{29}$ These findings suggest that RFA should be considered when developing a management strategy for bleeding superficial vascular lesions refractory to the standard APC therapy.

\section{TOPICAL THERAPY}

Mechanical and thermal therapies are effective, especially when the bleeding source is localized, and the endoscopist is able to direct focal therapy effectively. However, for difficult-to-reach and diffuse bleeding, achieving hemostasis can be challenging with the aforementioned devices. Currently, there are two topical agents licensed for the treatment of NVUGIB. Hemospray (Cook Medical, Limerik, Ireland) and Endoclot (EPI, Santa Clara, CA, USA) have demonstrated 
promising results in achieving hemostasis in NVUGIB. These agents have benign safety profiles and are easy to administer, making them appealing options when the conventional mechanical, thermal, and injection therapies are unsuccessful.

Endoclot, which is available in Australia and Europe, is a starch-derived modified polysaccharide suspended as hydrophilic particles, which can be sprayed through the instrument channel onto a bleeding source. The substance is propelled through the application catheter with the assistance of an air compressor. Once in contact with the bleeding area, the polysaccharide polymers absorb water, rendering the remaining blood hyper-concentrated for platelets and clotting factors, resulting in an accelerated clotting cascade. The concentrated dehydrated blood product additionally creates a gel-like texture that adheres to the bleeding lesion, providing a seal. Owing to its starch-derived polysaccharide nature, the Endoclot is absorbed across the gastrointestinal mucosa, but undergoes rapid degradation. ${ }^{23}$ Data on the efficacy of Endoclot are limited to date; however, it has been shown to be effective in achieving hemostasis in post-procedural bleeding following 20 cases of colonic endoscopic mucosal resections. ${ }^{30}$

Hemospray is another commercially available topical hemostatic agent currently available outside of the United States. For years, inert mineral powders have been used by military surgeons for achieving hemostasis. Hemospray has been designed similarly as a proprietary mineral powder propelled through either 10-Fr or 7-Fr catheters with the assistance of a carbon dioxide pump. Upon contact with blood products, the mineral powder absorbs water from the blood, thereby forming a viscous mechanical barrier, in comparison with Endoclot, which is rich in clotting factors and platelets that drive the clotting cascade. ${ }^{31}$ The inert mineral powder is not absorbed, and at the time of second-look endoscopy on the following day, it can no longer be identified. ${ }^{32}$ In a multicenter study, Hemospray has been shown to achieve primary hemostasis as monotherapy in $85 \%(n=66)$ of patients who presented with upper gastrointestinal bleeding of various non-variceal etiologies. ${ }^{33}$ This degree of success was further substantiated by an additional investigation, which reported a primary hemostasis success rate of $98.5 \%$ in patients $(n=60)$ also presenting with NVUGIB. ${ }^{32}$ Hemospray may be useful in cases of diffuse mucosal tumor bleeding, as it has been shown to achieve durable hemostasis for bleeding from gastroduodenal malignancies. ${ }^{34}$

Overall, topical hemostatic agents are a promising option given their ease, safety, and relative efficacy compared with traditional approaches. These agents can be applied to a broad area; this allows management of lesions that may have multiple bleeding sites. ${ }^{35}$ Further studies regarding their comparative efficacy and safety need to be performed before deter- mining the exact role these topical agents will assume in the hierarchy of endoscopic hemostatic approaches and in various clinical scenarios.

\section{REVIEW OF ENDOSCOPIC APPROACHES TO THE COMMON CAUSES OF NVUGIB}

The abovementioned techniques can be applied in clinical practice to achieve hemostasis in patients presenting with upper gastrointestinal bleeding. The decision on which strategy to utilize should be driven by the underlying lesion responsible for acute bleeding. The most common etiologies of NVUGIB are peptic ulcer disease (20\%-50\%), esophagitis (5\%-15\%), gastroduodenal erosions ( $8 \%-15 \%)$, Mallory-Weiss tears $(8 \%-15 \%)$, and vascular malformations. ${ }^{36,37}$ PUB is the most common cause of NVUGIB; as such, it has been extensively evaluated with respect to the optimal hemostatic approach. A large meta-analysis including 75 studies has demonstrated that while thermal therapy and mechanical clip use are successful in achieving hemostasis, no single therapy has been proven to be superior to other therapies irrespective of the site of the lesion. ${ }^{5,38}$ As described previously, injection therapy with epinephrine alone is not adequate and should only be considered in conjunction with a more definitive therapy. With respect to non-variceal esophageal lesions that contribute to upper gastrointestinal bleeding, esophagitis and Mallory-Weiss tears are commonly encountered. Esophagitis secondary to gastroesophageal reflux disease, medications, radiation, or infection can present with upper gastrointestinal bleeding, and in the majority of patients, no endoscopic therapy is required. ${ }^{36}$ Conversely, Mallory-Weiss tears resulting from a laceration of the mucosa at the level of the gastroesophageal junction are more likely to require endoscopic therapy. Multipolar electrocautery is believed to be the most effective therapy for these lesions, while combination therapy of epinephrine injection and hemostatic clip use has also been found to be effective. ${ }^{39,40}$ To date, there are no prospective trials comparing the treatment methods for AUGIB caused by vascular malformations, such as GAVE and AVM. The utilization of co-aptive electrocautery, APC, or endoscopic ligation is considered effective. ${ }^{41}$ Dieulafoy lesions, which are submucosal arteries that can lead to gastrointestinal bleeding when exposed, can be found anywhere along the gastrointestinal tract, with the majority of which found within the stomach. While not a single approach has been shown to be superior, numerous endoscopic methods, such as banding, hemostatic clip placement, electrocautery, heater probe use, and laser therapy, have been shown to be effective in the management of bleeding Dieulafoy lesions. Similar to the management of 
PUB, injection of epinephrine or saline should only be considered in combination with a second hemostatic technique. ${ }^{42}$ While RFA was introduced earlier as an emerging strategy for the management of GAVE, APC has been the most commonly accepted and reported modality for the management of this vascular malformation. Following multiple sessions of APC, bleeding related to GAVE subsides, which is associated with a decreased need for further transfusion requirements. ${ }^{43}$ With respect to the location of bleeding lesions, there is no evidence that a single hemostatic technique is superior to others. However, from a technical aspect, electrocautery co-aptive techniques may be preferred for lesions that are difficult to appose, as they can be utilized in a tangential manner; conversely, the placement of hemostatic clips in this position can be challenging.

\section{CONCLUSIONS}

Endoscopic interventions continue to play a dominant role in the evaluation and management of AUGIB. While there has been a steady decline in the incidence of in-patient mortality due to NVUGIB, significant related morbidity, hospital length of stay, and need for blood transfusions continue to increase. ${ }^{2,3}$ To improve the rates of successful hemostasis, the tools available must be further improved, in addition to the endoscopist skills, and we must recognize the ideal clinical scenarios for their implementation. Recent advances in hemostatic clip designs have improved our ability to achieve more effective and durable mechanical hemostasis. The evolution of both contact and non-contact thermal therapies and incorporation of soft-coagulation hemostatic forceps and RFA have bolstered the armamentarium of tools for endoscopists to employ appropriate and successful therapy in a clinically individualized manner. Continued investigations on topical hemostatic sprays seem promising, and their future incorporation to our hierarchical approach to refractory, inaccessible, and diffuse bleeding can be expected.

\section{Conflicts of Interest}

The authors have no financial conflicts of interest.

\section{REFERENCES}

1. Hearnshaw SA, Logan RF, Lowe D, Travis SP, Murphy MF, Palmer KR. Acute upper gastrointestinal bleeding in the UK: patient characteristics, diagnoses and outcomes in the 2007 UK audit. Gut 2011;60:1327-1335.

2. Tielleman T, Bujanda D, Cryer B. Epidemiology and risk factors for upper gastrointestinal bleeding. Gastrointest Endosc Clin N Am 2015;25:415-428.
3. Abougergi MS, Travis AC, Saltzman JR. The in-hospital mortality rate for upper GI hemorrhage has decreased over 2 decades in the United States: a nationwide analysis. Gastrointest Endosc 2015;81:882-888.e1.

4. van Rensburg CJ, Cheer S. Pantoprazole for the treatment of peptic ulcer bleeding and prevention of rebleeding. Clin Med Insights Gastroenterol 2012;5:51-60.

5. Laine L, Jensen DM. Management of patients with ulcer bleeding. Am J Gastroenterol 2012;107:345-360; quiz 361.

6. Hwang JH, Fisher DA, Ben-Menachem T, et al. The role of endoscopy in the management of acute non-variceal upper GI bleeding. Gastrointest Endosc 2012;75:1132-1138.

7. Asge Technology Committee, Conway JD, Adler DG, et al. Endoscopic hemostatic devices. Gastrointest Endosc 2009;69:987-996.

8. Lin HJ, Hsieh YH, Tseng GY, Perng CL, Chang FY, Lee SD. A prospective, randomized trial of large- versus small-volume endoscopic injection of epinephrine for peptic ulcer bleeding. Gastrointest Endosc 2002;55:615-619.

9. Sung JJ, Tsoi KK, Lai LH, Wu JC, Lau JY. Endoscopic clipping versus injection and thermo-coagulation in the treatment of non-variceal upper gastrointestinal bleeding: a meta-analysis. Gut 2007;56:1364-1373.

10. Cook DJ, Guyatt GH, Salena BJ, Laine LA. Endoscopic therapy for acute nonvariceal upper gastrointestinal hemorrhage: a meta-analysis. Gastroenterology 1992;102:139-148.

11. Sacks HS, Chalmers TC, Blum AL, Berrier J, Pagano D. Endoscopic hemostasis. An effective therapy for bleeding peptic ulcers. JAMA 1990;264:494-499.

12. Kovacs TO, Jensen DM. Endoscopic therapy for severe ulcer bleeding. Gastrointest Endosc Clin N Am 2011;21:681-696.

13. Hachisu T, Miyazaki S, Hamaguchi K. Endoscopic clip-marking of lesions using the newly developed HX-3L clip. Surg Endosc 1989;3:142147.

14. Barkun AN, Martel M, Toubouti Y, Rahme E, Bardou M. Endoscopic hemostasis in peptic ulcer bleeding for patients with high-risk lesions: a series of meta-analyses. Gastrointest Endosc 2009;69:786-799.

15. Lin HJ, Lo WC, Cheng YC, Perng CL. Endoscopic hemoclip versus triclip placement in patients with high-risk peptic ulcer bleeding. Am J Gastroenterol 2007;102:539-543.

16. Kirschniak A, Kratt T, Stüker D, Braun A, Schurr MO, Konigsrainer A. A new endoscopic over-the-scope clip system for treatment of lesions and bleeding in the GI tract: first clinical experiences. Gastrointest Endosc 2007;66:162-167.

17. Naegel A, Bolz J, Zopf Y, et al. Hemodynamic efficacy of the over-thescope clip in an established porcine cadaveric model for spurting bleeding. Gastrointest Endosc 2012;75:152-159.

18. Manta R, Galloro G, Mangiavillano B, et al. Over-the-scope clip (OTSC) represents an effective endoscopic treatment for acute GI bleeding after failure of conventional techniques. Surg Endosc 2013;27:3162-3164.

19. Laine L. Therapeutic endoscopy and bleeding ulcers. Bipolar/multipolar electrocoagulation. Gastrointest Endosc 1990;36(5 Suppl):S38-S41.

20. Wang HM, Hsu PI, Lo GH, et al. Comparison of hemostatic efficacy for argon plasma coagulation and distilled water injection in treating highrisk bleeding ulcers. J Clin Gastroenterol 2009;43:941-945.

21. Chung SC, Leung JW, Sung JY, Lo KK, Li AK. Injection or heat probe for bleeding ulcer. Gastroenterology 1991;100:33-37.

22. Karaman A, Baskol M, Gursoy S, et al. Epinephrine plus argon plasma or heater probe coagulation in ulcer bleeding. World J Gastroenterol 2011;17:4109-4112.

23. Palmer R, Braden B. New and emerging endoscopic haemostasis techniques. Frontline Gastroenterol 2015;6:147-152.

24. Arima S, Sakata Y, Ogata S, et al. Evaluation of hemostasis with soft coagulation using endoscopic hemostatic forceps in comparison with metallic hemoclips for bleeding gastric ulcers: a prospective, randomized trial. J Gastroenterol 2010;45:501-505.

25. Nagata S, Kimura S, Ogoshi H, Hidaka T. Endoscopic hemostasis of gastric ulcer bleeding by hemostatic forceps coagulation. Dig Endosc 
2010;22 Suppl 1:S22-S25.

26. Fujishiro M, Abe N, Endo M, et al. Current managements and outcomes of peptic and artificial ulcer bleeding in Japan. Dig Endosc 2010;22 Suppl 1:S9-S14.

27. Nunoue T, Takenaka R, Hori K, et al. A randomized trial of monopolar soft-mode coagulation versus heater probe thermocoagulation for peptic ulcer bleeding. J Clin Gastroenterol 2015;49:472-476.

28. McGorisk T, Krishnan K, Keefer L, Komanduri S. Radiofrequency ablation for refractory gastric antral vascular ectasia (with video). Gastrointest Endosc 2013;78:584-588.

29. Dray X, Repici A, Gonzalez P, et al. Radiofrequency ablation for the treatment of gastric antral vascular ectasia. Endoscopy 2014;46:963-969.

30. Huang R, Pan Y, Hui N, et al. Polysaccharide hemostatic system for hemostasis management in colorectal endoscopic mucosal resection. Dig Endosc 2014;26:63-68.

31. ASGE Technology Committee, Song WK, Banerjee SLM, et al. Emerging technologies for endoscopic hemostasis. Gastrointest Endosc 2012;75:933-937.

32. Chen YI, Barkun A, Nolan S. Hemostatic powder TC-325 in the management of upper and lower gastrointestinal bleeding: a two-year experience at a single institution. Endoscopy 2015;47:167-171.

33. Smith LA, Stanley AJ, Bergman JJ, et al. Hemospray application in nonvariceal upper gastrointestinal bleeding: results of the survey to evaluate the application of hemospray in the luminal tract. J Clin Gastroenterol 2014;48:e89-e92.

34. Chen YI, Barkun AN, Soulellis C, Mayrand S, Ghali P. Use of the endoscopically applied hemostatic powder TC-325 in cancer-related upper GI hemorrhage: preliminary experience (with video). Gastrointest Endosc 2012;75:1278-1281.
35. Sung JJ, Luo D, Wu JC, et al. Early clinical experience of the safety and effectiveness of Hemospray in achieving hemostasis in patients with acute peptic ulcer bleeding. Endoscopy 2011;43:291-295.

36. Rockall TA, Logan RF, Devlin HB, Northfield TC. Incidence of and mortality from acute upper gastrointestinal haemorrhage in the United Kingdom. Steering committee and members of the national audit of acute upper gastrointestinal haemorrhage. BMJ 1995;311:222-226.

37. Boonpongmanee S, Fleischer DE, Pezzullo JC, et al. The frequency of peptic ulcer as a cause of upper-GI bleeding is exaggerated. Gastrointest Endosc 2004;59:788-794.

38. Laine L, McQuaid KR. Endoscopic therapy for bleeding ulcers: an evidence-based approach based on meta-analyses of randomized controlled trials. Clin Gastroenterol Hepatol 2009;7:33-47; quiz 1-2.

39. Gunay K, Cabioglu N, Barbaros U, Taviloglu K, Ertekin C. Endoscopic ligation for patients with active bleeding Mallory-Weiss tears. Surg Endosc 2001;15:1305-1307.

40. Huang SP, Wang HP, Lee YC, et al. Endoscopic hemoclip placement and epinephrine injection for Mallory-Weiss syndrome with active bleeding. Gastrointest Endosc 2002;55:842-846.

41. Wong RM, Ota S, Katoh A, et al. Endoscopic ligation for non-esophageal variceal upper gastrointestinal hemorrhage. Endoscopy 1998;30:774-777.

42. Norton ID, Petersen BT, Sorbi D, Balm RK, Alexander GL, Gostout CJ. Management and long-term prognosis of Dieulafoy lesion. Gastrointest Endosc 1999;50:762-767.

43. Kwan V, Bourke MJ, Williams SJ, et al. Argon plasma coagulation in the management of symptomatic gastrointestinal vascular lesions: experience in 100 consecutive patients with long-term follow-up. Am J Gastroenterol 2006;101:58-63. 